\title{
Trajectory Modification Using Elastic Force for Collision Avoidance of a Mobile Manipulator
}

\author{
Nak Yong Ko ${ }^{1}$, Reid G. Simmons ${ }^{2}$, and Dong Jin Seo ${ }^{1}$ \\ ${ }^{1}$ Dept. Control and Instrumentation Eng., Chosun Univ., Korea \\ nyko@chosun.ac.kr \\ 2 The Robotics Institute, Carnegie Mellon Univ., USA \\ reids@cs.cmu.edu
}

\begin{abstract}
This paper proposes a method for collision avoidance of a mobile manipulator. The method deals with the problem of driving a mobile manipulator from a starting configuration to a goal configuration avoiding obstacles. It modifies planned trajectory at every sampling time using elastic force and potential field force. It puts way poses throughout a planned trajectory, and the trajectory modification is accomplished by adjusting the way poses. The way poses are adjusted subject to the elastic force and the potential field force. The procedure repeats until the robot reaches its goal configuration through the way poses. This method results in smooth and adaptive trajectory in an environment with moving as well as stationary obstacles.
\end{abstract}

\section{Introduction}

This paper presents a collision avoidance method for a mobile manipulator. The method applies to the robots with nonholonomic mobile base as well as the holonomic one. It drives the robot from an initial configuration to a goal configuration avoiding obstacles which are moving as well as stationary. The pose and shape of the obstacles are assumed to be given in real time. In some cases the obstacles can be another robots or machines whose locations are known or monitored online. In other cases, some sensors such as scanning range sensors, sonars, or cameras can be used for obstacle detection.

Though it's hard to find a collision free trajectory for a mobile manipulator, there have been many well known approaches available for collision avoidance of a mobile base. The artificial potential field approach [1] and Vector Field Histogram [2] are popular methods for obstacle avoidance of mobile robots, and they have many variations for improvement [3]. While it is easy to apply and is widely used, it's local minimum problem requires some additional means to avoid trap or oscillatory situation. Another approach considering the path as the piecewise continuation of the circular segments $[4,5]$ can be applied to nonholonomic mobile bases as well as holonomic bases. Also the dynamic windows approaches [6,7] and the elastic band approach [8] well suit for obstacle avoidance of the mobile robots. The ASL method combined geometric search and elastic force approach. It also features concurrent motion planning and modification, 
and is used to guide a robot in crowded exhibition environment for extended periods [9].

Many robot control architectures usually separated motion planning and execution in two different control layers. In these frameworks, the motion planning level doesn't pay enough attention to the real time obstacle avoidance. As the computing ability improves, some methods incorporate motion planning and motion execution in one frame. Among them, the elastic strip method [10] modifies its planned motion for obstacle avoidance at every sampling time and traces the modified trajectory plan. Still, the elastic strip method is computation intensive because it requires computation of sweep volume, elastic tunnel, and checking the overlap of the elastic tunnel with obstacles. In some cases, a nonholonomic mobile base still cannot trace some of the planned trajectory because of its limited mobility.

The method proposed in this paper uses elastic force and potential field force, and is applicable to mobile manipulators with nonholonomic mobile base as well as holonomic one. The elastic force differs from usual attraction force toward goal pose. The elastic force not only attracts the robot toward the goal pose, it also keeps the trajectory continuous and smooth toward the goal pose. The potential field force deforms the trajectory for obstacle avoidance.

Unlike the Elastic Strip method, it doesn't use geometric calculation which has adverse effect on real time application. The method sets way poses between the initial pose and goal pose throughout the trajectory at predefined intervals. The robot follows through the way poses in sequence until it reaches to the goal pose. The section 2 explains the procedure of the method in detail. The section 3 shows some simulation results as well as a real implementation example. Section 4 concludes the paper with some discussions on the method.

\section{Collision Avoidance Procedure}

At every sampling time, the method modifies the trajectory planned at the previous sampling time. The modification of the trajectory means the modification of the way poses. The procedure is as follows.

1. Set an initial trajectory and way poses along the trajectory.

2. Modify the trajectory by modifying the way poses using the following procedure.

(a) Calculate the elastic force and repulsive potential field force exerting to every control points on the way poses.

(b) Calculate the torque or force on the joints of the robot in the way poses due to the force acting on the control points.

(c) Calculate the deformation angle or distance of each joint due to the torque or force on the joint.

(d) Change the joint rotation angle or translational distance by the deformation angle or distance calculated in the step ( 2c), and establish the new way poses. 
3. Trace the trajectory through the way poses.

4. Check if the robot reaches to the goal pose. If the robot reaches close enough to the goal pose then stop the procedure. If not, go to the step 2 .

The following sections explain the procedure in detail.

\subsection{Initial Trajectory and Way Pose}

The method uses way poses for trajectory modification. It sets an initial trajectory, and modifies the trajectory at every sampling time. The trajectory is considered as an elastic thread connecting the starting pose to goal pose. Between the initial pose to goal pose we set a number of way poses, and virtual elastic string connects each point on the robot body from the initial pose to the final goal pose via the corresponding points on each way pose. Fig. 1 depicts the initial pose, final pose, way poses, and the elastic connections between them.

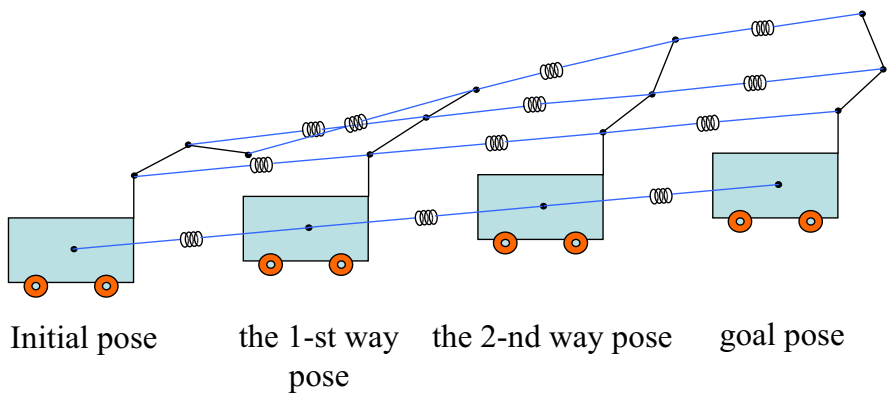

Fig. 1. Initial pose, final goal pose and way poses for a trajectory of a mobile manipulator

Though the path changes its shape subject to the elastic force and repulsive force, the topological property of the path relative to the location of the obstacles never change, because the path changes continuously and the path will neither be cut off nor be reconnected. If we set more way poses along the path, then the robot motion will become smoother. However, the increased number of way poses requires more calculations because the algorithm modifies every way pose at each sampling time. On the contrary, even though the way poses don't overlap with the obstacles, the robot can collide with some obstacles if consecutive way poses are not set close enough.

\subsection{Elastic Force and Repulsive Force Acting on a Control Point}

On the robot, some points called the control points are set. At the control points exert the elastic force and repulsive force. Though, it is the most desirable to apply and spread the elastic force and repulsive force at all the points throughout the body of the robot, only several points should be chosen for practical application. The control points should be chosen so that the effect of the forces 
on them can be as equivalent as possible to the effect of the forces spreading throughout the whole body of the robot.

In our application, we use the robot Bullwinkle shown in the Fig. 2(a) for verification of the method. The Bullwinkle uses skid-steering for mobility of the base, and has a manipulator with five revolute joints. Fig. 2(b) shows the dimension of the base. The values of the kinematic parameters of the manipulator are $d_{1}=223 \mathrm{~mm}, d_{4}=275 \mathrm{~mm}, a_{1}=63.5 \mathrm{~mm}, a_{2}=290 \mathrm{~mm}, a_{3}=$ $252.7 \mathrm{~mm}, a_{4}=49.23 \mathrm{~mm}$. On the Fig. 3, the control point ${ }^{j} \mathbf{p}_{i}$ represents the $i$-th control point in the $j$-th way pose. The superscript represents the order of the way pose, and the subscript represents the order of the control point in the way pose.

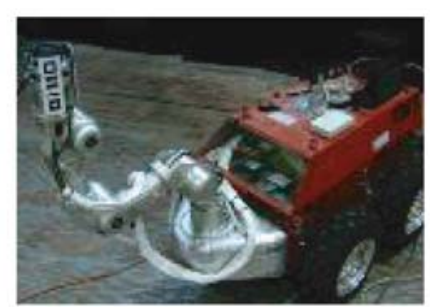

(a) The robot Bullwinkle has skidsteered mobile base and five joint manipulator

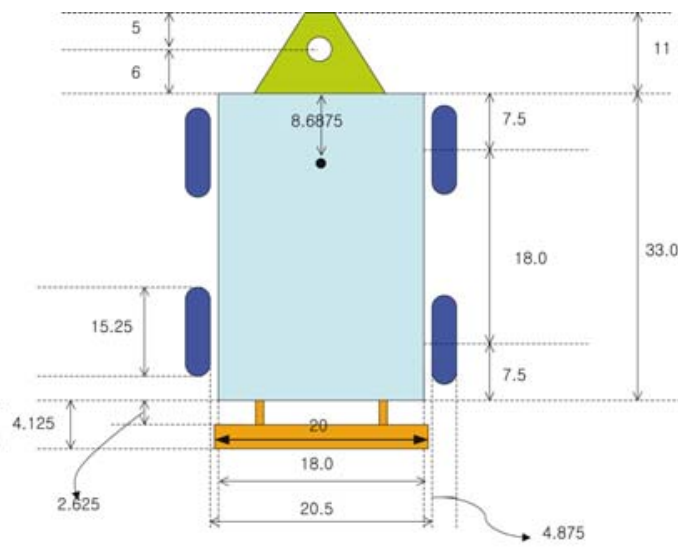

(b) Dimension of the base in inches

Fig. 2. The robot Bullwinkle and dimension of the base

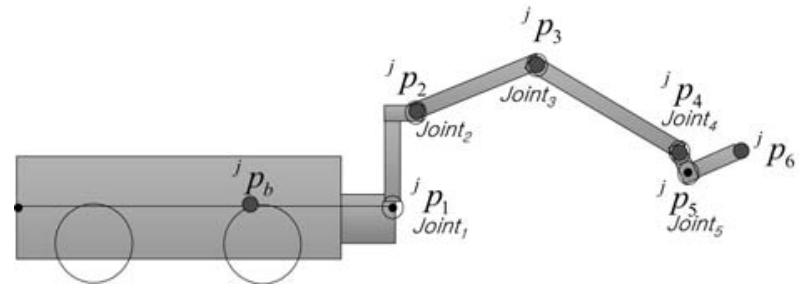

Fig. 3. Mobile manipulator Bullwinkle and the control points on the body. ${ }^{j} \mathbf{p}_{i}$ represents the $i$-th control point on the robot in its $j$-th way pose.

The elastic force ${ }^{j} \mathbf{e f}_{i}$ exerting to the control point ${ }^{j} \mathbf{p}_{i}$ is as follows.

$$
{ }^{j} \mathbf{e f}_{i}=k_{c}\left(\frac{d_{j-1, j}}{d_{j-1, j+1}}\left({ }^{j+1} \mathbf{p}_{i}-{ }^{j-1} \mathbf{p}_{i}\right)-\left({ }^{j} \mathbf{p}_{i}-{ }^{j-1} \mathbf{p}_{i}\right)\right)
$$


In the equation, the vectors ${ }^{j-1} \mathbf{p}_{i},{ }^{j} \mathbf{p}_{i}$, and ${ }^{j+1} \mathbf{p}_{i}$ represent the location of the $i$-th control point in its $(j-1)$-th, $j$-th, and $(j+1)$-th way pose respectively. $d_{j-1, j}$ is the distance between ${ }^{j-1} \mathbf{p}_{i}$ and ${ }^{j} \mathbf{p}_{i} . d_{j-1, j+1}$ is the distance from ${ }^{j-1} \mathbf{p}_{i}$ to ${ }^{j+1} \mathbf{p}_{i}$ via ${ }^{j} \mathbf{p}_{i}$. The elastic force tends to align the way poses from the initial to final location as straight as possible. Though each control point is likely to align straight throughout the way poses, the kinematic structure of the joint and link limits the straightness of the trajectory. The forces on the control points compromise and result in elastically stretched trajectory.

While the elastic force connects the initial pose to the final pose through way poses, repulsive force also works to push the trajectory away from obstacles. In our application, force by artificial potential field is used as the repulsive force. The following shows the repulsive potential field $V_{\text {rep }}\left({ }^{j} \mathbf{p}_{i}\right)$ and repulsive force ${ }^{j} \mathbf{r f}_{i}$ at the control point ${ }^{j} \mathbf{p}_{i}$.

$$
\begin{aligned}
& V_{\text {rep }}\left({ }^{j} \mathbf{p}_{i}\right)= \begin{cases}\frac{1}{2} k_{r}\left(d_{r}-d\left({ }^{j} \mathbf{p}_{i}\right)\right)^{2}, & \text { if } d\left({ }^{j} \mathbf{p}_{i}\right)<d_{r} \\
0, & \text { otherwise }\end{cases} \\
& { }^{j} \mathbf{r f}_{i}=-\nabla V_{\text {rep }}\left({ }^{j} \mathbf{p}_{i}\right)=k_{r}\left(d r-d\left({ }^{j} \mathbf{p}_{i}\right)\right) \frac{{ }^{j} \mathbf{p}_{i}-\mathbf{p}_{o}}{\left\|{ }^{j} \mathbf{p}_{i}-\mathbf{p}_{o}\right\|}
\end{aligned}
$$

In the equation, $d_{r}$ is the radius of the range under the influence of the repulsive force. $d\left({ }^{j} \mathbf{p}_{i}\right)$ is the shortest distance between the control point ${ }^{j} \mathbf{p}_{i}$ and the location $\mathbf{p}_{o}$ of an obstacle relevant to collision. $k_{r}$ is the coefficient for the repulsive force to the distance. The net force ${ }^{j} \mathbf{f}_{i}$ exerting on the control point ${ }^{j} \mathbf{p}_{i}$ is the sum of the elastic force and the repulsive force.

$$
{ }^{j} \mathbf{f}_{i}={ }^{j} \mathbf{e f}_{i}+{ }^{j} \mathbf{r f}_{i}, \quad \text { for } i=1,2, \ldots, 6
$$

For notational simplicity, in the following sections, we deleted the superscript $j$ which denotes a way pose if we only need to distinguish control points in a way pose. If there is no superscript in notations, it is supposed that all the control points are located in a way pose, for example in the $j$-th way pose.

\subsection{Force and Torque Adjusting the Way Pose}

The modification of the trajectory is accomplished by deforming the way poses. The deformation of the way pose means change of the configuration variables. The configuration variables for the base are $\left\{x_{b}, y_{b}, \theta_{b}\right\}$, and those for manipulator are $\left\{\theta_{1}, \theta_{2}, \ldots, \theta_{5}\right\}$. The rotational or translational deformation of the robot configuration is proportional to the joint torque or joint force. The joint torque or force is due to the force and torque on the control points generated by the elastic force and potential field force.

To find the joint torque acting on the joints, we use the Jacobian of the manipulator.

$$
\tau_{i}=\mathbf{J}_{i}^{T} \mathbf{f}_{i+1}
$$

Here, the vector $\tau_{i}=\left[\tau_{1}, \tau_{2}, \ldots, \tau_{i}\right]^{T}$ consists of the torques on the joints $1,2, \ldots, i$ and the vector $\mathbf{f}_{i+1}=\left[f_{i+1, x}, f_{i+1, y}, f_{i+1, z}\right]^{T}$ represents the force acting on the 
control point $\mathbf{p}_{i+1}$. The vector $\mathbf{f}_{i+1}$ is the sum of the elastic force and repulsive force acting on the control point $\mathbf{p}_{i+1}$. The matrix $\mathbf{J}_{i}$ is the $3 \times i$ Jacobian matrix satisfying the fellowing relationship,

$$
\dot{\mathbf{p}}_{i+1}=\mathbf{J}_{i} \dot{\mathbf{q}}_{i}
$$

where $\mathbf{p}_{i+1}=\left[p_{i+1, x}, p_{i+1, y}, p_{i+1, z}\right]^{T}$ is the position vector of the $(i+1)$-th control point and $\mathbf{q}_{i}=\left[\theta_{1}, \theta_{2}, \ldots, \theta_{i}\right]^{T}$ is the joint variable vector.

To find the torque due to the forces exerting on all the control points $\mathbf{p}_{1}, \ldots, \mathbf{p}_{6}$, it is required to sum all the torque vectors such that,

$$
\tau=\sum_{k=1}^{5}\left[\begin{array}{c}
\tau_{i} \\
\mathbf{0}
\end{array}\right]_{5 \times 1} .
$$

where $\tau=\left[\tau_{1}, \tau_{2}, \ldots, \tau_{5}\right]^{T}$ consists of all the five torques around the five manipulator joints.

For the mobile base, it is required to find the force and torque around the point $\mathbf{p}_{b}=\left[p_{b, x}, p_{b, y}, p_{b, z}\right]^{T}$. The force exerting on $\mathbf{p}_{b}$ is the sum of the elastic and repulsive force on the control points $\mathbf{p}_{i},(i=1,2, \ldots, 6)$ and the elastic and repulsive force at the control point $\mathbf{p}_{b}$ which is located on the mobile base itself. If we represent the elastic force and repulsive force on the point $\mathbf{p}_{b}$ as $\overline{\mathbf{f}}_{b}=\left[\bar{f}_{b, x}, \bar{f}_{b, y}, \bar{f}_{b, z}\right]^{T}$, then the net force $\mathbf{f}_{b}=\left[f_{b, x}, f_{b, y}, f_{b, z}\right]^{T}$ is found to be as the following.

$$
\mathbf{f}_{b}=\overline{\mathbf{f}}_{b}+\sum_{k=1}^{6} \mathbf{f}_{i}
$$

Here, we ignore the force in the $z$ direction since the mobile base doesn't have any mobility in the $z$ direction. The torque $\tau_{b}=\left[\tau_{b, x}, \tau_{b, y}, \tau_{b, z}\right]^{T}$ around $\mathbf{p}_{b}$ is due to the force on $\mathbf{p}_{i},(i=1,2, \ldots, 6)$, and it is expressed as the following.

$$
\tau_{b, x}=0, \tau_{b, y}=0, \tau_{b, z}=\sum_{k=1}^{6}\left(p_{i, x} f_{i, y}-p_{i, y} f_{i, x}\right)
$$

We ignore the torque around the direction $x$ and $y$ since the mobile base doesn't have any rotational mobility around the direction $x$ and $y$.

\subsection{Finding the Way Poses}

The way poses are updated every sampling time. The amount of deformation of the way pose for the manipulator is proportional to the force or torque applied to the joint. In our case, all the joints of the manipulator are revolute, so the rotation angles are found to be the followings.

$$
\delta \theta_{i}=\alpha_{i} \tau_{i},(i=1,2, \ldots, 5)
$$

In this equation, $\alpha_{i}$ is the coefficient for the rotation of the $i$-th joint to the joint torque $\tau_{i}$. For the mobile base, the rotation angle and displacement are as the followings.

$$
\delta x_{b}=\beta_{b, x} f_{b, x}, \delta y_{b}=\beta_{b, y} f_{b, y}, \delta \theta_{b}=\alpha_{b, z} \tau_{b, z}
$$


Here, $\beta_{b, x}$ and $\beta_{b, y}$ are the coefficients for the translation of the base to the force in the $x$-direction and $y$-direction respectively. $\alpha_{b, z}$ is the coefficient for the rotation of the base around the $z$-direction to the torque $\tau_{b, z}$.

\subsection{Tracing the Trajectory Through the Way Poses to the Goal Pose}

The way poses represent the trajectory of the motion. The method drives the robot through the way poses in sequence. Once the robot reaches a way pose, it then moves toward the next way pose. As the robot moves toward the goal, the number of way poses passed through increases and the number of remaining way poses waiting to be passed through decreases. This process repeats until the robot passes the last way pose and reaches the final goal pose.

For easy application, the robot is considered to be at its goal pose if each of the configuration variables falls within a range which is set around its goal pose value with clearance small enough. This is expressed in the following inequalities.

$$
\begin{gathered}
\left|x_{b}-x_{b, g}\right|<\varepsilon_{x, b},\left|y_{b}-y_{b, g}\right|<\varepsilon_{y, b},\left|\theta_{b}-\theta_{b, g}\right|<\varepsilon_{\theta, b} \\
\left|\theta_{i}-\theta_{i, g}\right|<\varepsilon_{\theta, i},(i=1,2, \ldots, 5)
\end{gathered}
$$

Here, $\left\{x_{b, g}, y_{b, g}, \theta_{b, g}\right\}$ and $\left\{\theta_{1, g}, \theta_{2, g}, \ldots, \theta_{5, g}\right\}$ are the goal pose of the base and manipulator respectively. $\varepsilon_{x, b}, \varepsilon_{y, b}, \varepsilon_{\theta, b}, \varepsilon_{\theta, i},(i=1,2, \ldots, 5)$ are the clearances for goal pose reach.

\section{Simulations and Experiments}

The proposed method is verified through simulations and an experimental implementation. The Fig. 4 and 5 show the results for simulations. In the Fig. 4(a),

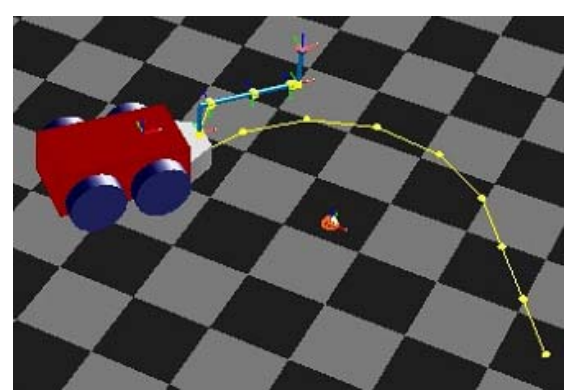

(a) Avoidance motion of the base

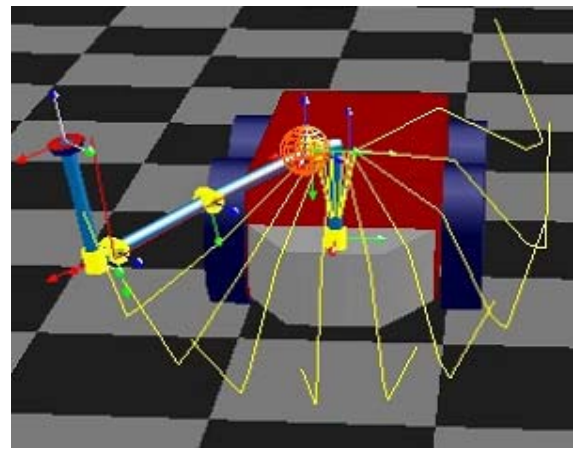

(b) Avoidance motion of the manipulator

Fig. 4. Simulation results. (a) Since the obstacle is on the floor, only the base motion is needed for collision avoidance. (b) The obstacle is far over the floor and the base doesn't need to care about collision. 


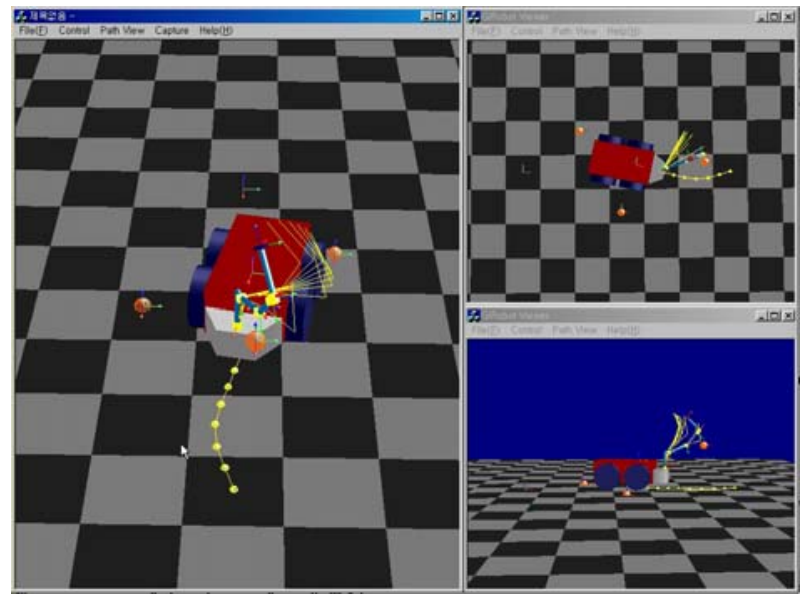

Fig. 5. Obstacle avoidance motion of the base and manipulator. The motion is captured in three different directions. There are three obstacles. Some of the obstacles affect the motion of the base as well as the manipulator.

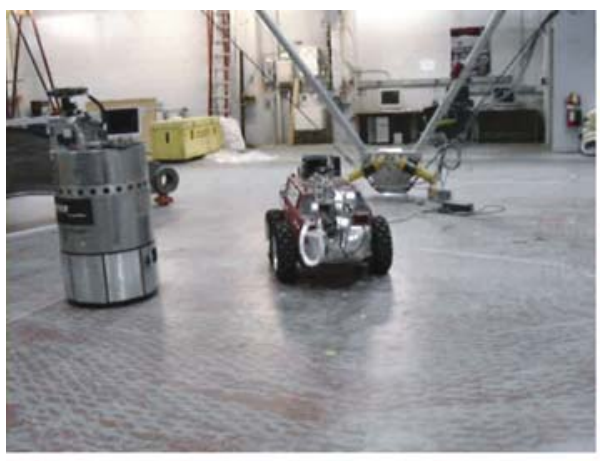

(a) Initial stage

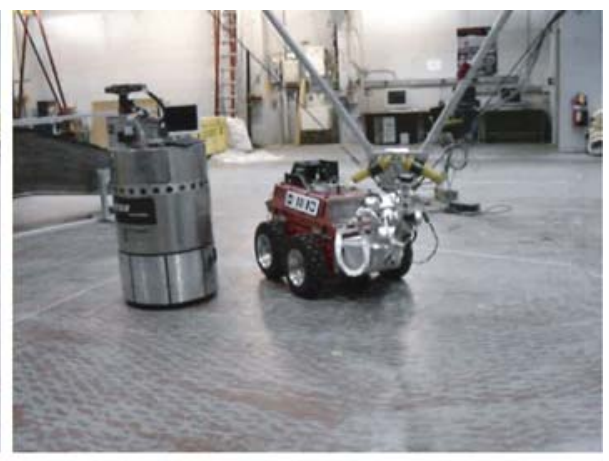

(b) Bullwinkle begins avoidance motion

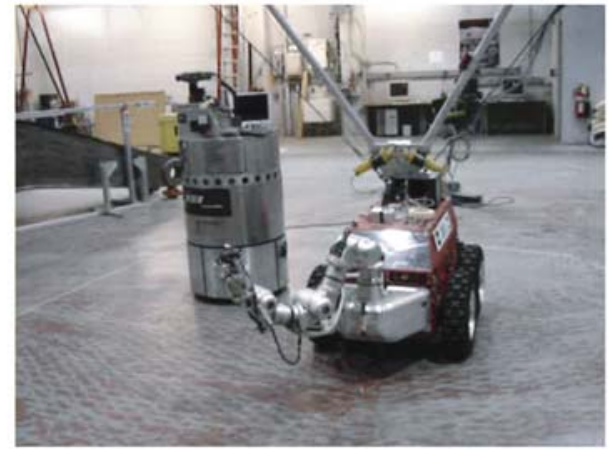

(c) Bullwinkle steers toward its goal after avoidance motion

Fig. 6. Result of the experiment. The Xavier is used as a moving obstacle. 
the obstacle is on the floor, and avoidance motion of only the base suffices collision avoidance. The manipulator is out of the range of potential field force from the obstacle. Likewise, in Fig. 4(b), the obstacle doesn't exert repulsive force to the base, and only the manipulator works for obstacle avoidance. Only the way poses of the arm lower down for obstacle avoidance under the repulsive potential field force.

In Fig. 5, there are 3 obstacles, and the mobile base as well as the manipulator works for obstacle avoidance. When the obstacles break into the queue of way poses, the way poses stretches out to avoid the obstacles. Later when the obstacles recede, the way poses restore back subject to the elastic force across the control points.

Fig. 6 shows the results of the experiment. The robot Xavier [11] is used as a moving obstacle. The Bullwinkle is commanded to go to a goal pose which is $5 \mathrm{~m}$ ahead of the initial heading. Xavier moves into the Bullwinkle's path from the upper right side of the Bullwinkle with the speed of $5 \mathrm{~cm} / \mathrm{s}$. For safety, the maximum speed of the Bullwinkle base is set to $10 \mathrm{~cm} / \mathrm{s}$.

\section{Conclusions}

A method for a mobile manipulator to avoid obstacles is described. The method uses elastic force and potential field force to adjust the trajectory for obstacle avoidance. The method has the following features.

1. The approach allows real time application even for mobile manipulator which has considerably high degrees of freedom. It results in smooth and efficient collision free trajectory for a mobile manipulator.

2. The method adjusts robot trajectory every sampling time, and avoids obstacles which are moving as well as stationary.

3. Though it uses potential field force for repulsion from obstacles, it also uses elastic force to keep in the robot shape. Therefore, the robot way poses restore and results in smooth and efficient trajectory toward the goal pose when obstacles move away.

For further improvement, the following should be addressed.

1. As the pose of the obstacles changes and the collision free path becomes very complicated, rerouting for simple path is necessary.

2. The number of way poses should be determined adaptively to the task and environment. If the obstacles are near the robot, it should be close, and if the obstacles are far away it can be sparse.

\section{Acknowledgement}

This study was supported by research funds from Chosun University, 2005. 


\section{References}

1. Khatib, O.: Real-time obstacle avoidance for manipulators and mobile robots. The Int. J. Robotics Research, Vol. 5, No. 1. (Spring 1986) 90-98

2. Boernstein, J., Koren, Y.: The vector field histogram-fast obstacle avoidance for mobile robots. IEEE Transaction on Robotics Automation, Vol. 7, No. 3. (June 1991) 278-288

3. Batavia, P., Nourbakhsh, I.: Path Planning for the Cye Personal Robot. Proc. International Conference in Intelligent Robots and Systems(IROS 2000), Vol. 1. (Oct. 2000) 15-20

4. Simmons, R.: The Curvature-Velocity Method for Local Obstacle Avoidance. Proc. 1996 IEEE International Conference on Robotics and Automation, Minneapolis MN (April 1996) 3375-3382

5. Ko, N. Y., Simmons, R.: The Lane-curvature Method for Local Obstacle Avoidance. Proc. International Conference in Intelligent Robots and Systems(IROS 1998), Victoria, B.C, Canada (Oct. 13-17 1998) 1615-1621

6. Brock, O., Khatib, O.: High-Speed Navigation Using the Global Dynamic Approach. Proc. of the IEEE Int. Conf. on Robotics and Automation, Vol. 1, (May 1999) 341-346

7. Fox, D., Burgard, W., Thrun,S.: The dynamic windows approach to collision avoidance. IEEE Robotics and automation magazine, 4(1) (March 1997)

8. Quinlan S., Khatib, O.: Elastic Bands: Connecting path planning and control. Proc. 1993 IEEE Int. Conf. on Robotics and Automation, Vol. 2. (1993) 802-807

9. Siegwart, R. et al.: Robox at Expo.02: A large scale Installation of Personal Robots. Journal of Robotics and Autonomous Systems, Vol. 42. (2003) 203-222

10. Brock, O. : Generating robot motion: The integration of planning and execution. PhD thesis, Stanford Univ. (1999)

11. http://www.cs.cmu.edu/ Xavier/ 